\section{REFERENCES}

1 Taylor DR, Bateman ED, Boulet LP, et al. A new perspective on concepts of asthma severity and control. Eur Respir J 2008; 32: 545-554.

2 Bush A, Hedlin G, Carlsen KH, et al. Severe childhood asthma: a common international approach? Lancet 2008; 372: 1019-1021.

3 Bracken M, Fleming L, Hall P, et al. Results of nurse-led home visits for children with difficult asthma. Thorax 2007; 62: Suppl. 111, A21-A22.

DOI: $10.1183 / 09031936.00177408$

From the authors:

We are grateful to F. Madsen, and A. Bush and colleagues for their positive comments on the work of the American Thoracic Society/European Respiratory Society Task Force on Asthma Control, Severity and Exacerbations, only part of which is contained in the recently published article [1]. We await the publication of the full statement, which contains specific recommendations about the assessment of asthma control.

We agree with F. Madsen that social and cultural perspectives on the part of the patient as well as the clinician will determine the relationship between optimum asthma control and the minimum treatment required to achieve it. In the paper we state that "the patient's perspective of what constitutes "ideal" control may reflect a personal balance of priorities between clinical benefits and real or perceived risks (including sideeffects and the cost of treatment)". This is consistent with F. Madsen's operational definition.

However, for reasons outlined in the article [1], it remains important that the relationship between treatment requirements and asthma severity should be considered separately. Asthma severity is defined as "the intensity of treatment required to control the patient's asthma'". This is focused more on objective rather than subjective measurements, and is affected by the asthma phenotype. The distinction may seem subtle, but it is an important one which needs to be grasped so that the relationship between clinical trial evidence and individual patient treatment requirements can be more clearly understood.

We believe that A. Bush and colleagues' model for "problematic severe asthma" does not differ substantially from that outlined in our paper (see fig. 1 in our previously published study [1]). Identifying therapy-resistant asthma as a particular phenotype separate from other causes of difficult-to-treat asthma is an important clinical goal that we would support. However, the model of A. Bush and colleagues does not take into account patients in whom good control is achieved using high doses of therapy, in which case it is severe but not necessarily clinically problematic.

\section{D.R. Taylor ${ }^{*}$, and H.K. Reddel ${ }^{\#, \oplus}$}

*Dunedin School of Medicine, University of Otago, Dunedin, New Zealand. "Woolcock Institute of Medical Research, Camperdown, Australia. "Co-chairs, American Thoracic Society/European Respiratory Society Task Force on Asthma Control, Severity and Exacerbations.

\section{STATEMENT OF INTEREST}

None declared.

\section{REFERENCES}

1 Taylor DR, Bateman ED, Boulet L-P, et al. A new perspective on concepts of asthma severity and control. Eur Respir J 2008; 32: $545-554$.

\title{
The clinical importance of rhinovirus-associated early wheezing
}

\section{To the Editors:}

In a recent report by a European Respiratory Society Task Force [1], human rhinovirus (HRV)-associated bronchiolitis and wheezing illness were not sufficiently discussed. Several recent observations highlight the importance of HRV infections in young wheezing children.

First, HRV is commonly associated with bronchiolitis and early-life wheezing, second only to respiratory syncytial virus (RSV). Detection rates have reached $40 \%$ in hospitalised wheezing infants [2, 3]. Moreover, HRV infection has been associated with the severity of illness [4].

Secondly, HRV infection among early wheezers is an important independent risk factor for recurrent wheezing [2, 5-8]. In population-based studies on young hospitalised children with acute wheezing, HRV infection has been associated with recurrent wheezing ( $\geqslant 3$ physician-confirmed episodes) during a 12-month follow-up period after the first episode (hazard ratio $5.1,95 \%$ confidence interval $(\mathrm{CI})$ 1.0-25, versus RSV-positive cases) and with the development of asthma at school-age (odds ratio 4.1, 95\% CI 1.0-17, versus HRV-negative cases) [2, 6].

In outpatient populations with increased risk for atopic illnesses, $\geqslant 1$ wheezing illness during infancy with HRV markedly increased the risk for third-year wheezing (odds ratio 10,95\% CI 4.1-26, versus HRV-positive cases with no moderate-to-severe respiratory infections) and modestly increased the risk for asthma at age 6 yrs (odds ratio 2.8, 95\% CI 1.1-7.5, versus HRVpositive cases with no wheezing) [5,8]. Interestingly at the third year of life, wheezing with HRV was markedly associated with asthma at age 6 yrs (odds ratio 26, 95\% CI 8.2-80, versus those 